\title{
Tsafon
}

Revue d'études juives du Nord

$74 \mid 2017$

La Déclaration Balfour, vers l'État d'Israël ?

\section{Nissim de Camondo (Le lieutenant), Correspondance et Journal de campagne, 1914- 1917}

Danielle Delmaire

\section{(2) OpenEdition}

1 Journals

Édition électronique

URL : https://journals.openedition.org/tsafon/438

DOI : $10.4000 /$ tsafon.438

ISSN : 2609-6420

Éditeur

Association Jean-Marie Delmaire

Édition imprimée

Date de publication : 1 décembre 2017

Pagination : 174-176

ISSN : 1149-6630

\section{Référence électronique}

Danielle Delmaire, « Nissim de Camondo (Le lieutenant), Correspondance et Journal de campagne, 19741917 », Tsafon [En ligne], 74 | 2017, mis en ligne le 31 mai 2018, consulté le 23 juin 2021. URL : http:// journals.openedition.org/tsafon/438; DOI : https://doi.org/10.4000/tsafon.438

Ce document a été généré automatiquement le 23 juin 2021.

Tsafon. Revues d'études juives du Nord 


\title{
Nissim de Camondo (Le lieutenant), Correspondance et Journal de
} campagne, 1914- 1917

\author{
Danielle Delmaire
}

\section{RÉFÉRENCE}

Nissim de Camondo (Le lieutenant), Correspondance et Journal de campagne, 1914- 1917,

Paris, Les Arts décoratifs, août 2017, 272 p., $39 €$.

1 Pour les amateurs de musées parisiens, le nom de Camondo évoque le musée Nissim de Camondo situé 63 rue Monceau à Paris. Ce luxueux hôtel fut la demeure de Moïse de Camondo, le père de Nissim (1892-1917), originaire de Constantinople mais de nationalité italienne, ce fut le roi d'Italie qui avait anobli la famille: Moïse comme Nissim étaient comte de Camondo. Et par sa mère, Nissim de Camondo est descendant des Cahen d'Anvers, eux-mêmes alliés à bien d'autres familles juives. «Le souvenir du lieutenant aviateur Nissim de Camondo résonnerait-il toujours dans les esprits si son père, accablé par sa mort tragique durant la Grande Guerre, n'avait pas légué son hôtel particulier et ses collections à l'État français » interroge l'historien Philippe Landau (p. 9). Après cette disparition, Moïse de Camondo n'a plus qu'une fille qui épouse en 1919 un membre de la famille Reinach nullement investi dans les activités bancaires et commerciales de son beau-père. La douleur du père est aggravée par l'appréhension de voir son nom et ses affaires disparaître. La disparition d'ailleurs sera totale 25 ans plus tard quand Béatrice de Camondo, épouse Reinach, son mari et ses deux enfants sont assassinés à Auschwitz. Ce musée est donc le seul et dernier vestige de la splendeur de la famille de Camondo, comme le constate P. Landau, qui appartient à «l'élite parisienne », la " grande bourgeoisie juive » pour reprendre les termes de Cyril Gange (lire plus haut le compte rendu de son livre). 
2 Outre un magnifique mobilier, le musée renferme, dans sa vingtaine de salles, des tableaux d'impressionnistes, des pièces d'art d'Extrême Orient, des objets de la Renaissance, du XVIII ${ }^{e}$ siècle français etc. C'est là aussi que sont conservées les archives de la famille dont la correspondance du lieutenant Nissim de Camondo durant la Première Guerre mondiale ainsi que son journal. Il faut être reconnaissant aux initiateurs de la publication car ils renseignent sur plusieurs fronts dans le nord de la France et en Belgique, sur la vie des officiers, sur le monde de la haute société juive.

3 Olivier Gabet, Sophie d'Aigneaux-Le Tarnec, Sylvie Legrand-Rossi, respectivement directeur et conservatrices au musée se sont associés à l'historien Philippe Landau pour présenter, en introduction, le musée, ses collections, Moïse et Nissim de Camondo et la correspondance. L'ouvrage est augmenté de photos de famille et du lieutenant sur la base d'aviation ainsi que des photos aériennes prises par Nissim, notamment du front de Verdun sur lesquelles on ne distingue que les zigzags des tranchées.

4 Les lettres sont éditées selon leur chronologie et, entre elles, s'insèrent les notes du journal. La plupart des lettres sont adressées à Moïse, surnommé « mon cher papa » ou " mon cher Piche » : une chaleureuse affection, paternelle et filiale, parcourt ces lettres. Nissim écrit aussi, mais moins souvent, à sa sœur Béatrice qui elle aussi est gratifiée de surnoms tendres, à quelques amis et le lot de lettres conservé au musée ne livre que de rares missives envoyées ou reçues de Renée Dorville, l'amante de Nissim. On peut expliquer la rareté de ces lettres, alors que Nissim était très épris, par le fait que Moïse appréciait peu cette liaison qui éloignait son fils de son milieu social et qu'il n'a pu récupérer que quelques lettres qui ne lui étaient pas destinées. S'y ajoutent quelques missives de Moïse à son fils, elles aussi sont rares car il est probable que Nissim ne les conserva pas alors que son père devait veiller attentivement à la préservation de la correspondance de son fils tant aimé.

5 L'échange épistolaire est très fréquent dans les premiers mois de la guerre : durant les cinq mois de l'année 1914, Nissim écrit autant que durant chacune des trois autres années. En 1914, il envoie quasiment une courte lettre quotidiennement. Par la suite, les échanges sont moins suivis et parfois Moïse semble en souffrir au point de s'en plaindre.

6 Nissim affectionne l'équitation, sport auquel son père l'a initié, sa sœur Béatrice est elle aussi une excellente cavalière. Il commence donc la guerre dans la cavalerie, ce qui l'expose à des combats dangereux mais l'éloigne plus ou moins des tranchées. Toutefois, au bout de plusieurs mois il est tenté par un autre sport et une autre armée : l'aviation. Il suit donc une formation qui l'enthousiasme et devient vite un excellent photographe des lignes ennemies à partir d'un avion, puis pilote des avions de combat. C'est lors d'un combat aérien qu'il disparaît en Lorraine le 5 septembre 1917. Moïse peut lire, dans les lettres des supérieurs de Nissim qui annoncent la disparition puis le décès de son fils, des qualificatifs élogieux. On y loue son courage, sa témérité et son sang-froid.

7 Cette correspondance renseigne sur divers épisodes de la Grande Guerre car Nissim participe à plusieurs batailles importantes. Il parcourt le Nord et le Pas-de-Calais en tous sens lors de la Course à la mer à la fin de 1914. Il raconte les errements des troupes et il a consigné, sur des cartes géographiques, les trajets suivis par l'armée française qui semble bien tourner en rond. En 1915, il est en Artois où les troupes piétinent, il raconte sobrement, pour ne pas effrayer son père probablement, les combats difficiles. En 1916, il survole Verdun, il prend des photos des zones sinistrées qui sont des 
témoins irréfutables de l'âpreté des batailles. Les éditeurs ont eu la bonne idée de les reproduire en pages 2 et 3 des couvertures.

8 Les lettres évoquent encore la souffrance au front bien que Nissim soit privilégié pour en être un peu plus éloigné que les soldats d'infanterie, grâce à son service dans la cavalerie puis dans l'aviation. Il supporte mal le froid et endure la privation de mets délicats auxquels son enfance choyée l'avait habitué. L'inconfort lui pèse également aussi sollicite-t-il fréquemment son père, voire sa sœur, pour améliorer l'ordinaire. Ses demandes sont parfois exigeantes : il commande des vêtements chauds, confortables, des bottes et des culottes de cheval mieux adaptées à ses exercices militaires. Il indique même les noms des artisans qui doivent les fabriquer. C'est la nourriture qu'il réclame avec le plus d'insistance : des gâteaux raffinés, des vins fins, du foie gras, et même du jambon! «Je voudrais qu'on m'envoie un jambon entier de chez Olida. S.V.P.», le 21 avril 1916, en pleine bataille de Verdun. Le lecteur peut s'étonner de la facilité avec laquelle son père trouve ces denrées qui, en cette période de guerre, doivent être rares et chères. Il demande aussi à recevoir de l'argent pour de menues dépenses qu'il ne décrit pas, sommes utiles éventuellement pour ses escapades loin du front. D'autres souhaits sont également surprenants comme recevoir un appareil photo (alors qu'il n'est pas encore photographe dans l'aviation) et surtout un phonographe. Ce ne sont pas seulement des désirs d'homme habitué au luxe car généreusement il partage avec ses camarades de combat les colis que son père s'est empressé de lui envoyer. Si Nissim peut paraitre exigeant, il se montre toujours très reconnaissant de la sollicitude paternelle. Ces demandes mettent en contraste l'ordinaire du soldat et l'abondance à laquelle un jeune comte, appartenant à l'élite sociale, est coutumier.

9 Lorsqu'il n'est pas mobilisé au front, le lieutenant de Camondo a la possibilité de s'échapper de l'enfer pour passer quelques heures, quelques jours, avec sa famille. Son père, sa sœur, qui ont les moyens de voyager dans la France en guerre, le rejoignent à l'arrière et font ensemble quelques virées, en voiture dans la campagne voire sur la côte normande. Avec d'autres gradés, il s'adonne à la chasse qu'il apprécie.

10 Toutefois, ces détails qui se lisent au fil des lettres masquent peut-être une réalité plus dure. Celle-ci transparaît entre les lignes lorsqu'il souhaite recevoir des objets plus ordinaires comme de simples chaussettes. Parfois, il s'attarde sur l'insalubrité des tranchées, de l'eau qui envahit tout. Mais constamment, Nissim s'efforce de ne pas inquiéter son père et minimise l'inconfort dont il souffre et surtout les dangers qui le menacent. Néanmoins, si l'on compare cette correspondance à d'autres signées par de simples soldats, on comprend que les tourments et les privations du front sont atténués lorsqu'on appartient à un corps d'officiers et à une famille aisée. N'appartenant pas à une famille aussi riche que celle des Camondo, l'historien Jules Isaac, juif lui aussi, ne livre pas le même récit sur sa vie de combattant. Il est vrai qu'il est plus âgé et que la famille, qu'il quitte pour combattre sa patrie, n'est pas un père mais une épouse éprise et des enfants en bas âge. En outre, il est vite hostile à la guerre qu'il trouve absurde ${ }^{7}$.

11 Le lecteur est encore informé sur l'état d'esprit d'un jeune homme, il n'a que 22 ans lorsque la guerre éclate, français d'abord, juif aussi. Longtemps dans ses lettres, il est convaincu de la supériorité des Français même s'il reconnaît que parfois les "Boches ", terme qu'il utilise abondamment, font preuve d'une résistance courageuse. Le 22 septembre 1914, il note dans son journal : "Escarmouche : 6 ou 7 boches caput » et le même jour il écrit à son père : «[...] les Allemands sont repoussés jusqu'à Noyon ». En ces premiers mois de guerre, son optimisme est solide : «Tout va bien; nous avançons 
tous les jours mais ce sera long " concède-t-il le 6 octobre 1914, dans sa lettre à son " papa chéri ». Plus tard, la durée de la guerre et la violence des combats amenuisent son enthousiasme, qui avec le recul de cent ans, peut paraître maintenant insouciant.

Il manifeste aussi de la compassion pour ses frères d'arme qui se battent à Verdun : « [...] le bombardement réciproque est toujours formidable et la situation des troupes aux tranchées est un enfer ", avoue-t-il le 28 avril 1916. Il apprécie encore le dévouement des troupes coloniales non sans une pointe de supériorité raciste, partagée par l'immense majorité des Français, à l'égard des Nord-Africains et Sénégalais.

13 C'est donc un journal et une correspondance qui fourmillent de détails sur la vie au front d'un jeune officier, patriote, dont l'inconfort est adouci par la générosité paternelle, sur les largesses d'une famille appartenant à l'élite sociale, sur l'affection qui lie un père à son fils et à sa fille.

\section{NOTES}

7. Jules Isaac fut le compagnon de Charles Péguy dans les années 1900-1914, avant que celui-ci tombe au combat. Sans doute aussi cette disparition précoce d'un ami très cher atteint Jules Isaac et développe en lui des sentiments pacifistes. Cf. Jules Isaac, Un historien dans la Grande Guerre. Lettres et carnets 1914-1917, Paris, Albin Michel, 2004. L'épouse et la fille de Jules Isaac ont disparu à Auschwitz, lui-même dut vivre clandestinement durant la Seconde Guerre mondiale. 